\title{
DIRAC PARTICLE IN AN AHARONOV-BOHM POTENTIAL: THE STRUCTURE OF THE FIRST ORDER S-MATRIX
}

\author{
M.S.SHIKAKHWA
}

\begin{abstract}
The structure of the interaction Hamiltonian in the first order $S$-matrix element of a Dirac particle in an Aharonov-Bohm (AB) field is analyzed and shown to have interesting algebraic properties. It is demonstrated that as a consequence of these properties, this interaction Hamiltonian splits both the incident and outgoing waves in the the first order $S$-matrix into their $\frac{\Sigma_{3}}{2}$-components ( eigenstates of the third component of the spin). The matrix element can then be viewed as the sum of two transitions taking place in these two channels of the spin. At the level of partial waves, each partial wave of the conserved total angular momentum is split into two partial waves of the orbital angular momentum in a manner consistent with the conservation of the total angular momentum quantum number.
\end{abstract}

Keywords: Aharonov-Bohm scattering, Scattering matrix

\section{INTRODUCTION}

The observation (Feinberg, 1963; Corinaldesi and Rafeli, 1978) that the first order Born amplitude of a non-relativistic spinless particle in an Aharonov-Bohm (AB) potential (Aharonov and Bohm, 1959) does not coincide with the exact amplitude when the latter is expanded to the same order triggered an unusual interest in the perturbative aspects of this problem. Various remedies for this essentially mathematical problem were suggested. Examples of some works that addressed this problem for non-relativistic spinless and spin one-half particles are Hagen (1995): Boz. Fainberg and Pak (1995): Ouvrv (1994); Manuel and Tarrach (1994); Ruiisenaars (1983); Romia and Shikakhwa (2004); Bergman and Lozano (1994); Hagen (1997); Fainberg. Pak and Shikakhwa (1998). For a Dirac particle, the success of the Born amplitude in providing results consistent with the exact amplitude expanded to the same order was demonstrated for the first order amplitude in Vera and Schmidt (1990), and for the second order in Boz and Pak (2000). A partial wave calculation of the first order amplitude was also recently reported (Shikakhwa and Pak, 2003) where consistency with the exact amplitude was also established.

The present work focuses on the interaction Hamiltonian in the first order $S$-matrix element of a Dirac particle. The algebraic properties of this interaction Hamiltonian reported partially for the first time in a cursory manner in Shikakhwa and Pak (2003) are exploited in depth and new interesting properties are reported. In particular, we note the major focus in the above work is on carrying out partial wave analysis of the first order Born amplitude of a Dirac particle in an AB potential using the basis of the conserved angular momentum operator. In the present work, on the other hand, the emphasis is not on the calculations, no amplitudes or so are 
reported. It is an analysis of the structure of the first order S-matrix in view of the algebraic properties of the interaction Hamiltonian. These analyses which are carried out without using any explicit wave functions enjoy an intrinsic importance by themselves and provide a new insight (as will be shown) into the structure of the first order transition in the $\mathrm{AB}$ potential. Verification of this structure using explicit wave functions is also given.

\section{Formalism}

A Dirac particle in an electromagnetic field is governed by the Hamiltonian $(\hbar=c=1)$ :

$$
H=H_{0}+H_{\text {int }}
$$

where

$$
H_{0}=\boldsymbol{\alpha} \cdot \mathbf{p}+\beta m
$$

and

$$
H_{\mathrm{int}}=e A_{0}-e \boldsymbol{\alpha} \cdot \mathbf{A}
$$

Here, $e$ is the charge of the particle, $\alpha_{i}=\beta \gamma_{i}$ and $\beta=\gamma_{4}$. The $\gamma^{\prime}$ s are the Dirac matrices: $\left\{\gamma_{\mu}, \gamma_{\nu}\right\}=2 g_{\mu \nu}$. While most of the treatment in this paper is independent of the explicit representation of these matrices, the Dirac-Pauli representation is used whenever called for:

$$
\gamma^{i}=\left(\begin{array}{cc}
0 & \sigma^{i} \\
-\sigma^{i} & 0
\end{array}\right), \quad \gamma^{4}=\left(\begin{array}{cc}
I & 0 \\
0 & -I
\end{array}\right)
$$

where $\sigma_{i}$ 's $(i=1, . ., 3)$ are the Pauli matrices, and $I$ is the $2 \times 2$ identity matrix. The first order $S$-matrix element for the particle is :

$$
S_{f i}^{(1)}=-i \int d^{4} x \bar{\psi}_{f}(x)\left(e \gamma_{\mu} A^{\mu}\right) \psi_{i}(x) .
$$

For the AB-potential (Aharonov and Bohm, 1959), we have:

$$
A_{0}=0,
$$

and

$$
\mathbf{A}=\frac{\Phi}{2 \pi \rho} \hat{\epsilon}_{\varphi}
$$

where $\rho=\sqrt{x^{2}+y^{2}}, \hat{\epsilon}_{\varphi}$ is the unit vector in the $\varphi$-direction, and $\Phi$ is the flux through the AB tube. The first order $S$-matrix in this case can then be written in the form:

$$
S_{f i}^{(1)}=i \alpha \int d \rho d \varphi \psi_{f}^{\dagger}(\mathbf{x})\left(O^{+}+O^{-}\right) \psi_{i}(\mathbf{x}) .
$$

Here, $\alpha=-e \Phi / 2 \pi$ (in perturbative calculations $0<\alpha<1$ ). The $z$ degree of freedom in the above matrix element was suppressed, since the $z$-component of the momentum of the particle-as is well-known-is conserved in the transition. This simplifies the treatment and does not introduce any loss of generality. An overall energy conserving $\delta$-function resulting from the time integration was also suppressed.The $O^{ \pm}$operators introduced above are defined as:

$$
O^{ \pm} \equiv\left(\frac{\alpha_{2} \pm i \alpha_{1}}{2}\right) e^{ \pm i \varphi}
$$


The above operators (note that $H_{\mathrm{int}}=\frac{\mathrm{O}^{+}+O^{-}}{\rho}$ )have interesting properties. One can easily check the following properties $\left(\Sigma_{i}=\frac{i}{2}\left[\gamma_{i}, \gamma_{j}\right] ; i, j=1 . .3\right)$ :

$$
\begin{aligned}
{\left[O^{+}, O^{-}\right] } & =-2\left(\frac{\Sigma_{3}}{2}\right) \\
\left\{O^{+}, O^{-}\right\} \equiv O^{+} O^{-}+O^{-} O^{+} & =I \\
\left(O^{ \pm}\right)^{\dagger} & =O^{\mp} \\
\left(O^{ \pm}\right)^{2} & =0
\end{aligned}
$$

Moreover, the algebra of $O^{ \pm}$with the third components of the spin, $\frac{\Sigma_{3}}{2}$, and the orbital angular momentum, $L_{3}$, is also interesting;

$$
\begin{aligned}
{\left[\frac{\Sigma_{3}}{2}, O^{ \pm}\right] } & =\mp O^{ \pm} \\
{\left[L_{3}, O^{ \pm}\right] } & = \pm O^{ \pm}=-\left[\frac{\Sigma_{3}}{2}, O^{ \pm}\right] \\
\left\{\frac{\Sigma_{3}}{2}, O^{ \pm}\right\} & =0 .
\end{aligned}
$$

Eqs. (14) and (15) suggest that the $O^{ \pm}$operators play the role of raising and lowering operators in the space of the eigenstates of $\frac{\Sigma_{3}}{2}$ and $L_{3}$. Now, products of the $O^{ \pm}$ operators give rise to the following two Hermitian operators:

$$
P^{ \pm} \equiv O^{\mp} O^{ \pm}
$$

which, in view of Eqs.(10) and (11) have the explicit forms

$$
P^{ \pm}=\frac{1}{2} \pm \frac{\Sigma_{3}}{2}
$$

These two operators have a series of interesting properties that follow from the set of equations, Eqs.(10 13)

$$
\begin{aligned}
\left(P^{ \pm}\right)^{2} & =P^{ \pm} \\
P^{+} P^{-}=P^{-} P^{+} & =0 \\
P^{+}+P^{-}=\left\{O^{+}, O^{-}\right\} & =I .
\end{aligned}
$$

Eq.(18), on the other hand leads to the following remarkable property of $P^{ \pm}$:

$$
\frac{\Sigma_{3}}{2} P^{ \pm}= \pm \frac{1}{2} P^{ \pm}
$$

The above properties suggest that $P^{ \pm}$is a projection operator. Indeed, for an arbitrary Dirac spinor $\Psi$, we have:

$$
\left(P^{+}+P^{-}\right) \Psi=P^{+} \Psi+P^{-} \Psi=\Psi
$$

with $P^{+} \Psi \quad\left(P^{-} \Psi\right)$ being, in view of Eq. (22), an eigenstate of $\frac{\Sigma_{3}}{2}$ with eigenvalue $+\frac{1}{2} \quad\left(-\frac{1}{2}\right)$.Thus, $P^{ \pm}$project out $\frac{\Sigma_{3}}{2}$ eigenstates with $\pm \frac{1}{2}$ eigenvalues out of an arbitrary $\Psi$, thus allowing the splitting of this $\Psi$ into a linear combination of these eigenstates. The following properties of the products of $P^{ \pm}$and $O^{ \pm}$follow by invoking Eq.(13):

$$
\begin{aligned}
P^{+} O^{+} & =P^{-} O^{-}=O^{+} P^{-}=O^{-} P^{+}=0 \\
O^{+} & =O^{+} P^{+}=P^{-} O^{+} P^{+} \\
O^{-} & =O^{-} P^{-}=P^{+} O^{-} P^{-} .
\end{aligned}
$$


The following brackets can also be easily verified:

$$
\begin{aligned}
{\left[O^{ \pm}, P^{+}\right] } & =\mp O^{ \pm} \\
{\left[O^{ \pm}, P^{-}\right] } & = \pm O^{ \pm}=-\left[O^{ \pm}, P^{+}\right] \\
{\left[\frac{\Sigma_{3}}{2}, P^{ \pm}\right] } & =\left[L_{3}, P^{ \pm}\right]=0 .
\end{aligned}
$$

Before leaving this section, we record the following major result, which is a consequence of Eqs.(11), (22), (25) and (26) :

$$
\frac{\Sigma_{3}}{2} O^{ \pm}=\mp \frac{1}{2} O^{ \pm}
$$

This relation says that $O^{ \pm}$acting on any arbitrary $\Psi$ project it onto an eigenstate of $\frac{\Sigma_{3}}{2}$ with eigenvalues $\mp \frac{1}{2}$.

\section{The First Order $S$-Martrix}

In this section, we will consider the consequences of the formalism developed above on the first order scattering matrix element. We start by writing Eq. (8) in the form

$$
S_{f i}^{(1)}=i \alpha M
$$

where

$$
\begin{aligned}
M & =\int d \rho d \varphi \psi_{f}^{\dagger}(x)\left(O^{+}+O^{-}\right) \psi_{i}(x) \\
& ={ }_{f}<\xi^{\prime}\left|\left(O^{+}+O^{-}\right)\right| \xi>_{i}
\end{aligned}
$$

In the second line of the above equation we have switched to the Dirac notation which will be adapted now on . The labels $\xi$ and $\xi^{\prime}$ denote the set of all quantum numbers of the initial and final states, respectively. This includes, for example, the quantum numbers of the free Hamiltonian $H_{0}$; a spin operator that commutes with $H_{0} \ldots$ etc. This set does not include the quantum numbers of $\frac{\Sigma_{3}}{2}$, as this does not commute with the free Hamiltonian and is not a constant of the transition. The amplitude $M$; Eq.(32), with the aid of Eqs. (25) and (26) can be written as

$$
M={ }_{f}<\xi^{\prime}\left|P^{-} O^{+} P^{+}\right| \xi>_{i}+_{f}<\xi^{\prime}\left|P^{+} O^{-} P^{-}\right| \xi>_{i}
$$

which, as a consequence of Eq.22) reduces to

$$
M={ }_{f}<\eta^{\prime}, \frac{-1}{2}\left|O^{+}\right| \eta, \frac{+1}{2}>_{i}+_{f}<\eta^{\prime}, \frac{+1}{2}\left|O^{-}\right| \eta, \frac{-1}{2}>_{i}
$$

The states $\mid \eta, \frac{ \pm 1}{2}>_{i}$ and $\mid \eta^{\prime}, \frac{ \pm 1}{2}>_{f}\left( \pm \frac{1}{2}\right.$ in the above states are the quantum numbers of $\frac{\Sigma_{3}}{2}$ ) are,respectively, the $\frac{\Sigma_{3}}{2}$-components of the initial and final free particle states. These are projected out of these states through the action of the operators $P^{ \pm}$in accordance with Eq.(22) . $\eta$ and $\eta^{\prime}$ are the set of all the other quantum numbers of the states, which are generally different from $\xi$ and $\xi^{\prime}$. The picture drawn by the above equation is that in the first order matrix element, the two operators $O^{ \pm}$constituting the effective interaction Hamiltonian split both the incident and outgoing waves into their $\frac{\Sigma_{3}}{2}$-components ( the two eigenstates of $\frac{\Sigma_{3}}{2}$ ) . The transition, is then the sum of two transitions induced by $O^{+}$and $O^{-}$, with $\mathrm{O}^{+}\left(\mathrm{O}^{-}\right)$linking only the $\frac{\Sigma_{3}}{2}$-component of the incident wave with eigenvalue $+\frac{1}{2}$ $\left(-\frac{1}{2}\right)$ to the $\frac{\Sigma_{3}}{2}$-component of the outgoing wave with eigenvalue $-\frac{1}{2}\left(+\frac{1}{2}\right)$.

Eq.(301), on the other hand, tells us that the states $O^{ \pm} \mid \eta, \pm \frac{1}{2}>_{i}$ are eigenstates of 
$\frac{\Sigma_{3}}{2}$ with eigenvalues $\mp \frac{1}{2}$. This means that the $O^{ \pm}$operators flip the spin of the $\frac{\Sigma_{3}}{2}$ states. Thus, we can define new states

$$
\left|\widehat{\eta, \mp \frac{1}{2}}\right\rangle_{i} \equiv O^{ \pm}\left|\eta, \pm \frac{1}{2}\right\rangle_{i}
$$

( note the change $\pm \frac{1}{2} \rightarrow \mp \frac{1}{2}$ in the $\frac{\Sigma_{3}}{2}$ quantum number). We then write the transition matrix $M$ as

$$
M={ }_{f}\left\langle\eta^{\prime}, \frac{-1}{2} \mid \eta \widehat{\frac{-1}{2}}\right\rangle_{i}+{ }_{f}\left\langle\eta^{\prime}, \frac{+1}{2} \mid \eta \widehat{\frac{+1}{2}}\right\rangle_{i} .
$$

The picture is even more interesting when one works with partial waves (Shikakhwa and Pak, 2003). Here, one expands the incident and outgoing plane waves of the $S$-matrix in terms of the $J$-waves that are eigenstates of the set of commuting operators: The total angular momentum operator $J_{3}=L_{3}+\frac{\Sigma_{3}}{2}$, the Hamiltonian $H_{0}$ and the spin operator $S_{3} \equiv \beta \Sigma_{3}$;

$$
\begin{aligned}
& H_{0}\left|E, j=l+\frac{1}{2}, s\right\rangle=E\left|E, j=l+\frac{1}{2}, s\right\rangle \\
& J_{3}\left|E, j=l+\frac{1}{2}, s\right\rangle=j_{3}\left|E, j=l+\frac{1}{2}, s\right\rangle=\left(\ell+\frac{1}{2}\right)\left|E, j=l+\frac{1}{2}, s\right\rangle, \quad \ell=0, \pm 1, \ldots \\
& S_{3}\left|E, j=l+\frac{1}{2}, s\right\rangle=s\left|E, j=l+\frac{1}{2}, s\right\rangle, \quad s= \pm 1,
\end{aligned}
$$

The $S$-matrix is then given as a sum over the partial amplitudes $M_{l}$ ( see section (4) below) :

$$
M_{l}=\left\langle E, j=l+\frac{1}{2}, s\left|O^{+}+O^{-}\right| E, j=l+\frac{1}{2}, s\right\rangle
$$

The conserved $J_{3}$ quantum number $j$ is set to $l+\frac{1}{2}, l=0 \pm 1, \pm 2, \ldots$. The quantum numbers $s$ also survives the transition as well as the corresponding operator is also conserved. The above $J$-waves which are energy eigenstates as well, are not eigenstates of $\frac{\Sigma_{3}}{2}$ nor of $L_{3}$. Eqs.(25) and (26), along with Eq.(22) have the following consequences:

$$
\begin{aligned}
& O^{+}\left|E, j=l+\frac{1}{2}, s\right\rangle=P^{-} O^{+}\left|\lambda, j=l+\frac{1}{2}, s ; l,+\frac{1}{2}\right\rangle \\
& O^{-}\left|E, j=l+\frac{1}{2}, s\right\rangle=P^{+} O^{-}\left|\lambda, j=l+\frac{1}{2}, s ; l+1,-\frac{1}{2}\right\rangle
\end{aligned}
$$

The states on the R.H.S are the components of the $J$-waves that are eigenstates of the operators $L_{3}$ and $\frac{\Sigma_{3}}{2}$ with eigenvalues $l, l+1$ and $\pm \frac{1}{2}$, respectively. We denote these with the $L$-waves. $\lambda$ is a collective index for any other possible quantum number; energy is not one of these, however. The quantum numbers of $\frac{\Sigma_{3}}{2}$ and $L_{3}$ in the above $L$-waves have been fixed by the use of Eq. (23), the fact that $j$ is conserved and fixed to $l+\frac{1}{2}$, and that $\left[J_{3}, L_{3}\right]=\left[S_{3}, L_{3}\right]=\left[J_{3}, \frac{\Sigma_{3}}{2}\right]=\left[S_{3}, \frac{\Sigma_{3}}{2}\right]=0$. 
Plugging Eqs.(39) and (40) into (38), and allowing $P^{ \pm}$to act to the left, we get

$$
\begin{aligned}
M_{l}= & \left\langle\lambda^{\prime}, j=l+\frac{1}{2}, s ; l+1,-\frac{1}{2}\left|O^{+}\right| \lambda, j=l+\frac{1}{2}, s ; l,+\frac{1}{2}\right\rangle \\
& +\left\langle\lambda^{\prime}, j=l+\frac{1}{2}, s ; l,+\frac{1}{2}\left|O^{-}\right| \lambda, j=l+\frac{1}{2}, s ; l+1,-\frac{1}{2}\right\rangle .
\end{aligned}
$$

The incident and outgoing $J$-waves are split by the interaction into their $L$-wave components, with $O^{+}\left(O^{-}\right)$linking incident $L$-waves with the set of eigenvalues $l,+\frac{1}{2}\left(l+1,-\frac{1}{2}\right)$ to outgoing $L$-waves with the set of eigenvalues $l+1,-\frac{1}{2}\left(l,+\frac{1}{2}\right)$. Note that the action of $O^{ \pm}$on the initial $L$-waves leads to raising or lowering of the quantum numbers of the operators $L_{3}$ and $\frac{\Sigma_{3}}{2}$ as dictated by Eqs. (14) and (15). Note also that this takes place in a manner that conserves the total angular momentum quantum number $j=l+\frac{1}{2}$. So, we can again define new states :

$$
\begin{aligned}
\left|\xi, j=l+\widehat{\frac{1}{2}, s ; l}+1,-\frac{1}{2}\right\rangle & \equiv O^{+}\left|\xi, j=l+\frac{1}{2}, s ; l,+\frac{1}{2}\right\rangle \\
\left|\xi, j=l+\frac{1}{2}, s ; l,+\frac{1}{2}\right\rangle & \equiv O^{-}\left|\xi, j=l+\frac{1}{2}, s ; l+1,-\frac{1}{2}\right\rangle .
\end{aligned}
$$

\section{The Matrix element Using Explicit Wave Functions}

In this section, explicit partial wave functions are going to be used to verify the results of the previous section. The incident and outgoing wave functions in the matrix element, Eq. (8), are expanded in terms of the eigen functions of the conserved total angular momentum operator $J_{3}$. These functions ( $J$-waves) that are simultaneous eigen functions of the set of operators $H_{0}, J_{3}$ and $S_{3}=\beta \Sigma_{3}$ in accordance with Eq.(37) have the explicit form (Shikakhwa and Pak, 2003):

$$
\psi_{\ell s}(\mathbf{x})=\frac{e^{i \ell \varphi}}{\sqrt{2 \pi} \sqrt{2 E} \sqrt{2 s}}\left(\begin{array}{c}
\sqrt{E+s m} \sqrt{s+1} J_{\ell}(\xi) \\
i \epsilon_{3} e^{i \varphi} \sqrt{E-s m} \sqrt{s-1} J_{\ell+1}(\xi) \\
\epsilon_{3} \sqrt{E+s m} \sqrt{s-1} J_{\ell}(\xi) \\
i e^{i \varphi} \sqrt{E-s m} \sqrt{s+1} J_{\ell+1}(\xi)
\end{array}\right)
$$

Here $J_{\ell}(\xi)$ is the Bessel function of order $\ell\left(\xi=p_{\perp} \rho\right)$, and $\epsilon_{3}=\operatorname{sgn}(s) . p_{\perp}$ is the magnitude of the planar momentum. The above eigen functions are normalized as

$$
\int d \rho d \varphi \psi_{\ell^{\prime} s^{\prime}}^{\dagger}(\mathbf{x}) \psi_{\ell s}(\mathbf{x})=\frac{1}{p_{\perp}} \delta\left(p_{\perp}-p_{\perp}^{\prime}\right) \delta_{\ell \ell^{\prime}} \delta_{s s^{\prime}} .
$$

If one takes $\psi_{i}(\mathbf{x})$ and $\psi_{f}(\mathbf{x})$ to be eigenstates of $S_{3}$ as well, then one can verify the following expansion of these in terms of the $\psi_{\ell s}(\mathbf{x})$ functions (Shikakhwa and Pak, 2003):

$$
\begin{aligned}
& \psi_{i}(\mathbf{x})=\sqrt{E_{i}} \sum_{\ell}(i)^{\ell} \psi_{\ell s}(\mathbf{x}) \\
& \psi_{f}(\mathbf{x})=\sqrt{E_{f}} \sum_{\ell}(i)^{\ell} e^{-i \ell \theta} \psi_{\ell s}(\mathbf{x}) .
\end{aligned}
$$

where $\theta$ is the scattering angle. So, the first order $S$-matrix element, Eq. (8), takes the form

$$
S_{f i}^{(1)}=i \alpha E \sum_{\ell}(i)^{\ell} \sum_{\ell^{\prime}}(-i)^{\ell^{\prime}} e^{i \ell^{\prime} \theta} M_{\ell}
$$


where $M_{\ell}$ is now given by

$$
M_{\ell}=\int d \rho d \varphi \psi_{\ell^{\prime} s^{\prime}}^{\dagger}(\mathbf{x})\left(O^{+}+O^{-}\right) \psi_{\ell s}(\mathbf{x})
$$

with $O^{ \pm}$as defined earlier. It is easy to check now that

$$
\begin{aligned}
\left(P^{+}+P^{-}\right) \psi_{\ell s}(\mathbf{x})=\psi_{\ell s}(\mathbf{x}) & =P^{+} \psi_{\ell s}(\mathbf{x})+P^{-} \psi_{\ell s}(\mathbf{x}) \\
& =\phi_{\ell,+\frac{1}{2}}(\mathbf{x})+\phi_{\ell+1,-\frac{1}{2}}(\mathbf{x})
\end{aligned}
$$

where the functions

$$
\begin{aligned}
\text { (50) } \phi_{\ell,+\frac{1}{2}}(\mathbf{x}) & =\frac{1}{\sqrt{2 \pi} \sqrt{2 E} \sqrt{2 s}}\left(\begin{array}{c}
\sqrt{E+s m} \sqrt{s+1} J_{\ell}(\xi) e^{i \ell \varphi} \\
0 \\
\epsilon_{3} \sqrt{E+s m} \sqrt{s-1} J_{\ell}(\xi) e^{i \ell \varphi} \\
0
\end{array}\right) \\
(51) \phi_{\ell+1,-\frac{1}{2}}(\mathbf{x}) & =\frac{1}{\sqrt{2 \pi} \sqrt{2 E} \sqrt{2 s}}\left(\begin{array}{c}
0 \\
i \epsilon_{3} \sqrt{E-s m} \sqrt{s-1} J_{\ell+1}(\xi) e^{i(\ell+1) \varphi} \\
0 \\
i \sqrt{E-s m} \sqrt{s+1} J_{\ell+1}(\xi) e^{i(\ell+1) \varphi}
\end{array}\right)
\end{aligned}
$$

are easily checked to be eigenstates of $\frac{\Sigma 3}{2}$ and $L_{3}$-as they should be- with eigenvalues $+\frac{1}{2}\left(-\frac{1}{2}\right)$ and $l(l+1)$, respectively. Thus, with the use of Eqs.(24 26) we have now $M_{\ell}$ as:

$$
M_{\ell}=\int d \rho d \varphi\left(\phi_{\ell+1,-\frac{1}{2}}^{\dagger}(\mathbf{x}) O^{+} \phi_{\ell,+\frac{1}{2}}(\mathbf{x})+\phi_{\ell,+\frac{1}{2}}^{\dagger}(\mathbf{x}) O^{-} \phi_{\ell+1,-\frac{1}{2}}(\mathbf{x})\right)
$$

which is just Eq. (41). Note that the primes on the quantum numbers have been dropped as the matrix element is diagonal in these quantum numbers since the corresponding operators $J_{3}$ and $S_{3}=\beta \Sigma_{3}$ are conserved. Now, following Eqs.(42) and (43), we define

$$
\begin{aligned}
& \widehat{\phi}_{\ell+1,-\frac{1}{2}}(\mathbf{x})=O^{+} \phi_{\ell,+\frac{1}{2}}(\mathbf{x})=\frac{1}{\sqrt{2 \pi} \sqrt{2 E} \sqrt{2 s}}\left(\begin{array}{c}
0 \\
i \epsilon_{3} \sqrt{E+s m} \sqrt{s-1} J_{\ell}(\xi) e^{i(\ell+1) \varphi} \\
0 \\
i \sqrt{E+s m} \sqrt{s+1} J_{\ell}(\xi) e^{i(\ell+1) \varphi}
\end{array}\right) \\
& \widehat{\phi}_{\ell,+\frac{1}{2}}(\mathbf{x})=O^{-} \phi_{\ell+1,-\frac{1}{2}}(\mathbf{x})=\frac{1}{\sqrt{E \pi} \sqrt{2 E} \sqrt{2 s}}\left(\begin{array}{c}
\sqrt{s+1} J_{\ell+1}(\xi) e^{i \ell \varphi} \\
0 \\
\epsilon_{3} \sqrt{E-s m} \sqrt{s-1} J_{\ell+1}(\xi) e^{i \ell \varphi} \\
0
\end{array}\right)
\end{aligned}
$$

It is a trivial task to check that $\widehat{\phi}_{\ell+1,-\frac{1}{2}}(\mathbf{x})$ and $\widehat{\phi}_{\ell,+\frac{1}{2}}(\mathbf{x})$ are eigenstates of $\frac{\Sigma 3}{2}$ and $L_{3}$ with the indicated eigenvalues. At this point, with the explicit wave functions at hand, it would be interesting to investigate the relation between the $L$-waves $\widehat{\phi}_{\ell,+\frac{1}{2}}(\mathbf{x})\left(\widehat{\phi}_{\ell+1,-\frac{1}{2}}(\mathbf{x})\right)$ and the incident $L$-waves $\phi_{\ell,+\frac{1}{2}}(\mathbf{x})\left(\phi_{\ell+1,-\frac{1}{2}}(\mathbf{x})\right)$. Comparing Eqs. (50) and (53), for example, we see that $\widehat{\phi}_{\ell,+\frac{1}{2}}(\mathbf{x})$ is -apart from a constantthe same as $\phi_{\ell,+\frac{1}{2}}(\mathbf{x})$ except for the order of the Bessel function which is shifted by +1 in the former. It is, on the other hand, shifted by -1 in $\widehat{\phi}_{\ell+1,-\frac{1}{2}}$ (x) in comparison to $\phi_{\ell+1,-\frac{1}{2}}(\mathbf{x})$. This shift in the order of the Bessel function gives rise to the phase shift of each partial amplitude. It was shown in Shikakhwa and Pak (2003) 
that the integral in the partial amplitudes $M_{\ell}$ reduces to an integral over Bessel functions of the form $\int d \rho J_{\ell+1} J_{\ell}$, and this integral gives the partial phase shifts. Thus, The operators $O^{ \pm}$, while flipping the spin and orbital angular momentum quantum numbers of each ingoing $L$-wave to match that of the outgoing one, keeps the order of the Bessel functions in the two waves shifted by \pm 1 so as to generate the the phase shifts in the scattering process. To put this on more formal grounds, we define the two operators $d^{ \pm}$:

$$
d^{ \pm} \equiv\left(\partial_{\rho} \pm \frac{i}{\rho} \partial_{\varphi}\right)=\left(\partial_{\rho} \mp \frac{L_{3}}{\rho}\right)
$$

that satisfy

$$
\left[d^{ \pm}, L_{3}\right]=\left[d^{ \pm}, \frac{\Sigma_{3}}{2}\right]=0 .
$$

We note that these operators - upon employing the well-known recurrence relations of Bessel functions- have the property

$$
\begin{aligned}
d^{+} J_{\ell}(\xi) e^{i \ell \varphi} & =-p_{\perp} J_{\ell+1}(\xi) e^{i \ell \varphi} \\
d^{-} J_{\ell+1}(\xi) e^{i(\ell+1) \varphi} & =p_{\perp} J_{\ell}(\xi) e^{i(\ell+1) \varphi}
\end{aligned}
$$

Therefore, we can now write:

$$
\begin{aligned}
& \widehat{\phi}_{\ell+1,-\frac{1}{2}}(\mathbf{x})=O^{+} \phi_{\ell,+\frac{1}{2}}(\mathbf{x})=\frac{1}{p_{\perp}} \sqrt{\frac{E+s m}{E-s m}} d^{-} \phi_{\ell+1,-\frac{1}{2}}(\mathbf{x}) \\
& \widehat{\phi}_{\ell,+\frac{1}{2}}(\mathbf{x})=O^{-} \phi_{\ell+1,-\frac{1}{2}}(\mathbf{x})=-\frac{1}{p_{\perp}} \sqrt{\frac{E-s m}{E+s m}} d^{+} \phi_{\ell,+\frac{1}{2}}(\mathbf{x})
\end{aligned}
$$

Substituting the above two equations into Eq.(152), we get :

$$
M_{\ell}=\frac{1}{p_{\perp}} \int d \rho d \varphi\left[\sqrt{\frac{E+s m}{E-s m}}\left(\phi_{\ell+1,-\frac{1}{2}}^{\dagger}(\mathbf{x}) d^{-} \phi_{\ell+1,-\frac{1}{2}}(\mathbf{x})\right)-\sqrt{\frac{E-s m}{E+s m}}\left(\phi_{\ell,+\frac{1}{2}}^{\dagger}(\mathbf{x}) d^{+} \phi_{\ell,+\frac{1}{2}}(\mathbf{x})\right)\right]
$$

The above equation says that we can write each partial amplitude as a sum of two transitions, with each one being a transition among two $L$-waves that have the same $L_{3}$ and $\frac{\Sigma_{3}}{2}$ quantum numbers. These $L$-waves are connected by the $d^{ \pm}$operators which merely affect the radial component of each partial wave, thus inducing the partial phase shifts. It is interesting indeed that in each of the two terms of the amplitude, the quantum numbers of the orbital angular momentum $L_{3}$ and the spin $\frac{\Sigma_{3}}{2}$ are conserved in the transition. It is as if we have replaced the $L_{3}$ and $\frac{\Sigma_{3}}{2}$ non-conserving interactions $O^{ \pm}$with the $d^{ \pm}$interactions that conserve these quantum numbers at the level of each partial $L$-wave.

\section{Concluding Remarks}

A new insight into the structure of the first order $S$-matrix of a Dirac particle in an $\mathrm{AB}$ potential was provided through the derivation of interesting algebraic properties of the interaction Hamiltonian appearing in the matrix element. It was shown that the interaction Hamiltonian is the sum of the two operators $O^{ \pm}$and algebraic properties of these and related operators were reported. The fact that these operators have the properties (Eqs. (25126) $) O^{ \pm}=P^{\mp} O^{ \pm} P^{ \pm}$along with the fact that $\frac{\Sigma_{3}}{2} P^{ \pm}= \pm \frac{1}{2} P^{ \pm}$(Eq. (22)), were shown to suggest the following picture of the transition matrix element: The interaction splits both the incident and outgoing 
plane waves into their (unconserved) $\frac{\Sigma_{3}}{2}$ components so that the matrix element is the sum of two transitions from two initial eigenstates of the $\frac{\Sigma_{3}}{2}$ operator to final eigenstates of the same operators . Each of the operators $O^{ \pm}$, by flipping the spin as a result of the property (Eq. (300) $\frac{\Sigma_{3}}{2} O^{ \pm}=\mp \frac{1}{2} O^{ \pm}$, links only the states with opposite spin quantum numbers. The transition, then, becomes the sum of two transitions induced by the two operators, each taking place in one of the spin channels. At the level of partial waves, the transition in each partial amplitude is the sum of two transitions taking place among the two unconserved $L$-wave components of each incident and outgoing $J$-wave.The fact that the total angular momentum of each partial wave is conserved leads to a flip not only of the $\frac{\Sigma_{3}}{2}$ quantum number, but also of the $L_{3}$ quantum number in the transition. Working with explicit partial wave functions, we have shown that it is possible to write each partial amplitude as the sum of two transitions, induced by a couple of two new operators, $d^{ \pm}$, taking place among $L$-partial wave functions such that the $\frac{\Sigma_{3}}{2}$ and $L_{3}$ quantum numbers are conserved in each of these transitions.

Finally, note that in deriving Eq. (34), no assumptions were made regarding the spin operator that one chooses to diagonalize with the free Hamiltonian. In the case of partial wave analysis (Eq.(41)), however, the spin operator was taken to be $S_{3}=\beta \Sigma_{3}$. The result, however, depends only on the fact that this spin operator commutes with the operators $O^{ \pm}$, i.e is a constant of the transition. Any other spin operator having this property will lead to the same result.

\section{Acknowledgments}

The author is indebted to professor N.K.Pak and Dr. B.Tekin for a critical reading of an earlier version of the manuscript, and for very helpful discussions. He also thanks the physics department of Middle East Technical University at Ankara where this work was initiated for hospitality. Partial financial support by The Scientific and Technical Research Council of Turkey (TÜBÍTAK) is also acknowledged.

\section{REFERENCES}

Aharonov, Y. and Bohm,D. (1959), Physical Review 115,485.

Bergman, O. and Lozano, G. (1994), Ann.Phys.229,416.

Boz, M. and Pak, N.K. (2000), Phys.Rev.D 62,045022.

Boz, M., Fainberg, V.Ya. and Pak, N.K. (1995), Phys. Lett. A 207,1.

Corinaldesi, E. and Rafeli, F. (1978), Am. J. Phys.46, 1185.

Fainberg, V.Ya., Pak, N.K. and Shikakhwa, M.S.(1995), J.Phys. A 31,3531.

Feinberg, E. L. (1963), Sov. Phys. Usp.5, 753 .

Hagen, C.R. (1995), Phys. Rev.D52,2466.

Hagen, C.R. (1997), Phys. Rev.D56,2250.

Manuel, C. and Tarrach, R. (1994), Phys. Lett. B 328,113.

Ouvry, S. (1994), Phys. Rev.D52,5296.

Romia, Y. and Shikakhwa, M. (2004), Tur.J.Phys.28,73.

Ruijsenaars, R. (1983), Ann.Phys.46,1.

Shikakhwa, M.S. and Pak, N.K. (2003), Phys. Rev.D67,105019.

Vera, F. and Schmidt, I. (1990), Phys. Rev.D42,3591.

Department of Physics, University of Jordan, 11942-Amman, Jordan

E-mail address: moody@ju.edu.jo 